\title{
Design of Speech Denoising Algorithm Based on Wavelet Threshold Function and PSO
}

\author{
Lanyong ZHANG ${ }^{\mathrm{a}}$, Ruixuan ZHANG $^{\mathrm{a} 1}$ and Papavassiliou CHRISTOS ${ }^{\mathrm{b}}$ \\ ${ }^{a}$ College of Intelligent Systems Science and Engineering, Harbin Engineering \\ University, Harbin, China \\ ${ }^{b}$ Department of Electrical and Electronic Engineering, Imperial College London, \\ London, United Kingdom
}

\begin{abstract}
At present, there are many shortcomings in the discontinuity of wavelet threshold function and the constant threshold of different decomposition layers and the constant error it produced. The amplitude-frequency characteristics of wavelet filters are studied and analyzed by mathematical modeling. An improved wavelet threshold function with adjustable parameters is proposed. Particle swarm optimization (PSO) algorithm is used to find the optimal parameters of the improved threshold function in a background noise environment. The improved wavelet threshold function is combined with Bayesian threshold method to obtain the threshold based on Bayesian criterion, which makes the threshold adaptive in different layers and overcomes the shortcomings of fixed threshold. Finally, the speech signal with optimal wavelet coefficients is obtained after reconstruction. Compared with the traditional threshold function, Simulation results show that the improved threshold function achieves precise notch denoising, effectively retains the singularity and eigenvalues of the signal, and reduces the signal distortion.
\end{abstract}

Keywords. Speech enhancement, wavelet transform, threshold function, bayesian threshold

\section{Introduction}

Noise in speech signal is mainly generated in the process of signal acquisition and transmission, and the ultimate purpose of noise elimination is to get more acceptable speech signal from noisy signal. Nowadays, many denoising algorithms have been proposed[1]. At present, wavelet technology and its improved algorithms are widely used in speech recognition and mobile communications. References [2] proposed a method to improve the threshold function in order to overcome the shortcomings of the wavelet transform soft and hard threshold method. References [3] The improved method only has a certain effect on periodic signals, and the effect scene is relatively single.

There are other algorithms, such as Spectral Subtraction (SS), Wiener Filter(WF), Least Mean Square algorithm (LMS), Subspace Method(SM), Deep Neural Networks(DNN) and so on. There are various problems in these methods, Therefore, scholars have proposed improved methods or new methods[4-7], such as wavelet

\footnotetext{
${ }^{1}$ Corresponding Author, Ruixuan Zhang, College of Intelligent Systems Science and Engineering, Harbin Engineering University, Harbin, China; Email: 13643292271@163.com
} 
transform enhancement algorithm is one of them[8]. Aiming at the deficiencies of traditional denoising methods that the threshold is fixed, this paper proposes a method of combining wavelet threshold function with adjustable parameters and Bayesian threshold is proposed, The particle swarm optimization algorithm is used to find the optimal adjustment parameters[9], which makes it adaptive at different levels and can remove clutter more effectively, Finally, simulation experiments verify the effectiveness of the new algorithm.

\section{Wavelet Threshold Function Denoising}

\subsection{Denoising Principle of Wavelet Threshold Function}

For a noise signal $\mathrm{y}(\mathrm{n})$ with length $\mathrm{N}$, the model can be expressed as:

$$
y(n)=x(n)+e(n)
$$

Among them, $y(n)$ is the contaminated signal, $x(n)$ represents a pure signal and $e(n)$ is a noise signal[10].

Following are the basic steps of multi-scale wavelet denoising[11]:

(1) For the noisy signal, first set a critical threshold, as a comparison value for comparison with wavelet coefficients, the multi-scale wavelet decomposition of the noisy signal is carried out by choosing the appropriate wavelet base and the number of decomposition layers, so that the wavelet coefficients of each layer can be obtained.

(2) Choose appropriate threshold and threshold function for each layer of high frequency wavelet system, and get the wavelet coefficients after each processing.

(3) The enhanced speech signal can be obtained by reconstructing the high-frequency and low-frequency wavelet coefficients of each layer.

\subsection{Improved threshold function}

From the analysis of the denoising steps of the wavelet threshold function, we can see that the denoising effect is related to the threshold function, the noise threshold of each layer and the reconstruction accuracy, therefore, the improvement of the threshold function is the key content of people's research. There are two common methods of traditional threshold function: soft threshold function and hard threshold function[12-13]. The hard threshold function is expressed as:

$$
y_{j, k}= \begin{cases}w_{j, k} & \left|w_{j, k}\right|>\lambda \\ 0 & \left|w_{j, k}\right| \leq \lambda\end{cases}
$$

When the wavelet coefficients are larger than the threshold value, the basic characteristics of the original initial signal are preserved to a certain extent. Since the threshold function is discontinuous at the threshold $\pm \lambda$, the pseudo-Gibbs phenomenon will easily occur when the wavelet coefficients are reconstructed after denoising.

Soft threshold function:

$$
\mathrm{y}_{\mathrm{j}, \mathrm{k}}= \begin{cases}\operatorname{sgn}\left(\omega_{\mathrm{j}, \mathrm{k}}\right)^{*}\left(\mathrm{w}_{\mathrm{j}, \mathrm{K}}-\lambda\right) & \left|\mathrm{w}_{\mathrm{j}, \mathrm{k}}\right|>\lambda \\ 0 & \left|\mathrm{w}_{\mathrm{j}, \mathrm{k}}\right| \leq \lambda\end{cases}
$$


Formula (3) shows that there is a constant difference between the wavelet coefficients $w_{j, k}$ of noisy signals and the output wavelet coefficients $\mathrm{y}_{\mathrm{j}, \mathrm{k}}$. Compared with the hard threshold function, the soft threshold function is continuous at $\pm \lambda$, but the reconstruction accuracy of the soft threshold function is not enough, this results in the loss of some useful speech signals. From the above analysis, we can see that both hard threshold function and soft threshold function have some shortcomings. The expression of improved wavelet threshold function in reference [14] is as follows:

$y_{j, k}= \begin{cases}\operatorname{sgn}\left(w_{j, k}\right) *\left(w_{j, k}-\lambda * k \sqrt{w_{j, k}^{2}-\lambda^{2}}\right) & \left|w_{j, k}\right|>\lambda \\ \operatorname{sng}\left(w_{j, k}\right) * k * \frac{w_{j, k}^{2}}{\lambda} & \left|w_{j, k}\right| \leq \lambda\end{cases}$

Among them, $\lambda$ is the threshold of each decomposition wavelet coefficients, $\mathrm{k}$ is the adjustment parameter. From the proposed threshold function formula, we can see that the change of the threshold function is between the soft and hard threshold functions, and the denoising effect is better than the traditional method to a certain extent. The modulus transformation of coefficients after wavelet transform conforms to the exponential attenuation characteristic. When the threshold value is lower than the threshold value, the threshold function does not satisfy the exponential form. This will inevitably result in unsatisfactory denoising and speech distortion, thus affecting the quality of reconstructed speech signal.

Reference [15] pointed out that the wavelet threshold function is based on an improved non-negative dead zone threshold function:

$y_{j, k}=\left\{\begin{array}{cc}\operatorname{sgn}\left(w_{j, k}-\frac{\lambda^{2}}{2\left|w_{j, k}\right|}\right) * e^{2\left(\lambda-\left|w_{j, k}\right|\right)} & \left|w_{j, k}\right|>\lambda \\ \operatorname{sgn}\left(w_{j, k}\right) * \frac{\lambda\left(\left.e^{8 \mid w_{j, k}}\right|_{-e^{8 p}}\right)}{2\left(e^{8 \lambda}-e^{8 p}\right)} & \left|w_{j, k}\right| \leq \lambda\end{array}\right.$

In the formula $\mathrm{p} \in(0, \lambda)$, although the improved threshold function solves the shortcoming that the wavelet coefficients are directly zero when the non-negative threshold function is less than the threshold value, when the wavelet decomposition coefficients are larger than the threshold value, the threshold function is basically fixed when the input noise intensity is different, which will cause distortion to the enhanced speech quality.

Considering that soft and hard threshold functions are odd functions, combining the above two ideas of threshold functions. In this paper, an improved method based on exponential function is proposed, and the exponents with different bases are set at the threshold of absolute value $\lambda$. This method can better denoise the wavelet coefficients of each layer of noisy signal and greatly improve the effect of denoising. The following is the improved expression of threshold function: the place is also continuous, and the following is the improved expression of threshold function:

$y_{j, k}=\left\{\begin{array}{l}\operatorname{sgn}\left(w_{j, k}\right) *\left(w_{j, k}-\frac{\lambda}{1+\alpha} * \gamma \sqrt{\sqrt{w_{j, k}^{2}-\lambda^{2}}}\right)\left|w_{j, k}\right|>\lambda \\ \operatorname{sgn}\left(w_{j, k}\right) * \frac{\alpha}{1+\alpha} * e^{10^{*}\left(\left|w_{j, k}\right|-\lambda\right)} *\left|w_{j, k}\right|\left|w_{j, k}\right| \leq \lambda\end{array}\right.$ 
In order to prove the continuity of the improved threshold function, since the improved threshold function is also an odd function, it is only necessary to prove that it is continuous in the right half of the definition domain.

When $\left|\mathrm{w}_{\mathrm{j}, \mathrm{k}}\right| \rightarrow \lambda:$

$\lim _{w_{j, k \rightarrow \lambda^{+}}} \operatorname{sgn}\left(w_{j, k}\right) *\left(\left|w_{j, k}\right|-\frac{\lambda}{1+\alpha} * \lambda \sqrt{w_{j, k}^{2}-\lambda^{2}}\right)=\frac{\alpha}{1+\alpha} * \lambda$
$\lim _{w_{j, k \rightarrow \lambda^{-}}} \operatorname{sgn}\left(w_{j, k}\right) * \frac{\alpha}{1+\alpha} * e^{10\left(\left|w_{j, k}\right|-\lambda\right)} *\left|w_{j, k}\right|=\frac{\alpha}{1+\alpha} * \lambda$

From Formulas (7) and (8), we can see $\lim _{\mathrm{w}_{\mathrm{j}, \mathrm{k} \rightarrow \lambda^{+}}}=\lim _{\mathrm{w}_{\mathrm{j}, \mathrm{k} \rightarrow \lambda^{-}}}=\frac{\alpha}{1+\alpha} * \lambda$, So we can know that the threshold function proposed at $\lambda$ is continuous. Similarly, we can know that the function at $-\lambda$ is also continuous.

When $\left|w_{j, k}\right|>\lambda$, The output signal $w_{\mathrm{j}, \mathrm{k}}$ is between $\left|\mathrm{w}_{\mathrm{j}, \mathrm{k}}\right|^{*} \operatorname{sgn}\left(\mathrm{w}_{\mathrm{j}, \mathrm{k}}\right)$. This is because we take any real number for $\alpha, 0<\frac{1}{1+\alpha} \leq 1$, And Formula $\sqrt{\sqrt{\mathrm{w}_{\mathrm{j}, \mathrm{k}}^{2}-\lambda^{2}}}$ knows, when $\gamma \in(0,1)$, The value of arbitrary $\omega_{\mathrm{j}, \mathrm{k}}$, the values are between $(0,1)$, when $\omega_{\mathrm{j}, \mathrm{k}} \rightarrow \infty$, $\gamma^{\sqrt{\omega_{\mathrm{j}, \mathrm{k}}^{2}-\lambda^{2}}}$ approaches zero. therefore $\mathrm{y}_{\mathrm{j}, \mathrm{k}} \rightarrow \omega_{\mathrm{j}, \mathrm{k}}$, In this way, the new threshold function does not have the problem of fixed deviation value relative to the soft threshold function.

\subsection{Threshold selection}

In the wavelet threshold de-noising algorithm, the acquisition of threshold value lambda also determines the effect of de-noising. If the value of $\lambda$ is too large, some useful signals in speech signal will be removed in the process of de-noising, which will easily lead to speech distortion. On the contrary, if the value of $\lambda$ is too small, there will be more residual noise components in de-noising, resulting in unsatisfactory de-noising effect[16]. In order to reduce the threshold of different layers, it is necessary to be able to adaptively follow the increase of wavelet decomposition scale. So the Bayesian threshold is used to acquire the noise threshold, and the following process is calculated concretely:

$\mathrm{r}(\mathrm{T})=\overline{\mathrm{E}}(\overline{\mathrm{x}}-\mathrm{x})^{2}=\mathrm{Ex}_{\mathrm{x}} \mathrm{E}_{\mathrm{x} \mid \mathrm{y}}(\overline{\mathrm{x}}-\mathrm{x})^{2}=\iint(n(y)-x)^{2} P(y \mid x) P(x) d y d x=\delta^{2} \omega\left(\frac{\delta_{x}^{2}}{\delta^{2}}, \frac{T}{\delta}\right)$

Among,

$\omega\left(\delta_{x}^{2}, \mathrm{~T}\right)=\delta_{x}^{2}+2\left(T^{2}+1-\delta_{x}^{2}\right) \bar{\phi}\left(\frac{T}{\sqrt{1+\delta_{x}^{2}}}\right)-2 T\left(1+\delta_{x}^{2}\right) * \phi\left(T, 1+\delta_{x}^{2}\right)$

Density function:

$$
\begin{gathered}
\phi\left(\mathrm{x}, \delta_{x}^{2}\right)=\frac{1}{\sqrt{2 \pi \delta^{2}}} \exp \left(-\frac{x^{2}}{2 \delta^{2}}\right) \\
\bar{\phi}(x)=\int_{x}^{\infty} \phi(t, 1) d t
\end{gathered}
$$

Threshold calculation expression:

$$
T_{j}=\frac{\delta^{2}}{\delta_{x}}
$$


In the above formula, $\delta^{2}$ is the noise variance, $\delta_{\mathrm{x}}$ is the standard deviation of subband coefficients, and $\mathrm{j}$ represents a layer in the stratification.For formula (13), the estimates proposed by Donoho and Johnstone are used to calculate $\delta^{2}$.

$$
\bar{\delta}=\frac{\operatorname{median}\left(\left|y_{j, k}\right|\right)}{0.6745}
$$

$\mathrm{y}$ (i) in the formula represents the wavelet coefficients of different layers.

$$
\bar{\delta}=\frac{1}{n} \sum_{i=1}^{n} y_{j, k}^{2}
$$

Among them, $\mathrm{n}$ represents the length of each decomposition wavelet coefficients, which can be seen from $\delta_{\mathrm{y}}^{2}=\delta_{\mathrm{x}}^{2}+\delta^{2}$ :

$$
\overline{\delta_{x}}=\sqrt{\max \left(\delta_{y}^{2}-\delta^{2}, 0\right)}
$$

Therefore, the threshold based on Bayesian criterion can be obtained by (14) 15 (16). It can be seen that the Bayesian threshold has self-adaptability in different layers and overcomes the shortcomings of fixed threshold, can better realize the wavelet threshold function denoising.

\section{Parameter acquisition of improved threshold function based on particle swarm optimization}

\subsection{Wavelet Denoising Fitness Function}

In this paper, an improved threshold function is proposed. There are two adjusting parameters, namely, lambda and alpha. In the face of different input signal noise ratios, in order to improve the threshold function, the adjusting parameters can be changed to adapt to different input signal-to-noise ratios. Particle swarm optimization (PSO) is used as a tool to find the optimal adjustment parameters. The PSO algorithm is a simulation of the social information sharing mechanism of a group of birds foraging for food. Each particle in the PSO algorithm is a solution in the solution space. It adjusts its flight according to the flight experience of itself and its companions. Get the optimal solution in the current solution space. Taking output signal-to-noise ratio (SNR) as fitness function, the larger the output signal-to-noise ratio, the better the denoising effect[17]. The SNR criteria are defined as follows (17):

$$
\mathrm{SNR}=10 \lg \frac{\sum_{i=1}^{N} x(i)^{2}}{\sum_{i=1}^{N}(y(i)-x(i))^{2}}
$$

Among, $\mathrm{x}$ (i) denoised signal, y (i) denoised input signal and $\mathrm{N}$ denoised input signal.

\subsection{PSO algorithm and Optimizing Threshold Function to Adjust Parameter Estimation}

PSO algorithm initializes a group of particles as a feasible solution. The best position that each particle passes through is the optimal solution found by the particle itself, which is called the individual extreme value, and the best position that the entire group passes through is the optimal solution found for the entire group, which is called the global 
extreme value[18]. The following formula is that particles update their own speed and position through individual and group extremes.

$$
\begin{aligned}
v(\mathrm{t}+1)= & \mathrm{v}(\mathrm{t})+c_{1} * \operatorname{rand}() *\left[p_{i}(\mathrm{t})-x_{i}(\mathrm{t})\right]+c_{2} * \operatorname{rand}() *\left[\operatorname{zbest}(t)-x_{i}(t)\right] \quad(1 \leq i \leq N) \\
& p_{i}(t)=p_{i}(t)+0.5 * v(t)
\end{aligned}
$$

Threshold function is continuous and differentiable, which is beneficial to speech signal denoising. In this paper, particle swarm optimization algorithm is used to determine the optimal adjustment parameters of threshold function. The steps of denoising are as follows:

(1) Four wavelet coefficients of noisy speech signals are obtained by four-layer wavelet transform. The wavelet coefficients of different layers are used as the input of particle swarm optimization, where the four groups of lengths are N/2, N/4, N/8 and N/16 respectively.

(2) Setting some parameters of particle swarm optimization algorithm to initialize, the learning factor $\mathrm{C} 1=1.50, \mathrm{C} 2=1.50$, the number of particles $\mathrm{N}=20$, limiting the speed of particles to a range of [-0.50.5], the number of iterations to 300 , and the value range of particles to [0.10];

(3) The fitness values of all particles in the first iteration are calculated according to the fitness function expressed in formula (17). The maximum fitness values of individuals and groups are determined from the initial fitness values of particles, which are expressed by gbest and zbest values respectively.

(4) According to formula (18) (19), the velocity and fitness values of each particle in each generation are updated and determined to be within a given range. Then, the fitness values of the next generation of particles are updated according to the fitness function SNR.

(5) Renewal of the optimum values gbest and global optimum zbest in individual particles.

(6) If the maximum number of iterations is met, the update will be stopped, otherwise the update will continue to step (4).

(7) The optimal value required for output is zbest, which is obtained by the last stable iteration.

(8) The optimal adjustment parameters are obtained, which are substituted into the improved threshold function and combined with Bayesian threshold to denoise, and then the denoised wavelet coefficients are reconstructed to obtain the enhanced speech signal.

\section{Simulation results and analysis of experiments}

This paper uses MATLAB to process noisy speech signals with different input SNR. The pure speech signal used in the experiment is a SP01 English speech. The content is Birch can slide on smooth boards. The length of the data is 22529. Background noise is white, dining room noise and pink noise, respectively. Voice and noise are taken from NOIZEUS database and Noisex-92 standard noise database. The input SNR is set according to the different logarithmic power ratio of the noise signal to the pure speech signal of the same time length. The selected SNR of the noisy speech is $-10 \mathrm{~dB},-5 \mathrm{~dB}$, $0 \mathrm{~dB}, 5 \mathrm{~dB}, 10 \mathrm{~dB}$ and $15 \mathrm{~dB}$, respectively. Speech and noise sampling frequencies are $8000 \mathrm{~Hz}$, single channel, sampling bit is 16 bit. Here, sym6 is chosen as the wavelet basis function. The signal decomposition scale is $n=4$. Documents $[7]([13],[15])$ and the 
improved threshold function method are used to process the noisy signal. The following two tables are speech enhancement data under different threshold functions.

Figure 1 shows the curves of output SNR. From the output SNR curves, it can be seen that the improved threshold function has better denoising effect than other thresholding functions under the same input noise conditions, especially in the low signal-to-noise ratio of $-10 \mathrm{~dB} 5 \mathrm{~dB}$, the SNR increases more than $1 \mathrm{~dB}$, and it is also improved in other ranges. The curve of output RMSE shown in Figure 2 shows that the proposed threshold function has higher stability and smaller changes. These two curves show that the improved threshold function has better noise reduction effect, stronger antiinterference and less distortion of speech.

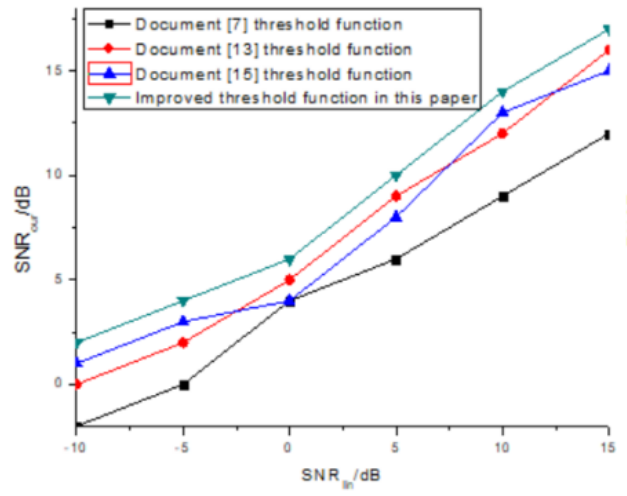

Figure 1.Output SNR Effect Contrast Curve

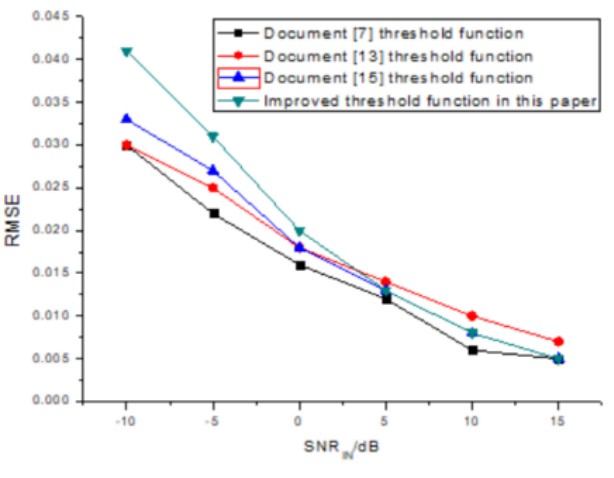

Figure 2.Output RMSE Effect Contrast Curve

Table.1 The LSD obtained with various threshold function in different backgrounds

\begin{tabular}{crrrrr}
\hline Noise & $\begin{array}{c}\text { SNR } \\
\text { dB }\end{array}$ & Document[7] & Document[13] & Document[15] & $\begin{array}{c}\text { Improved } \\
\text { threshold } \\
\text { function }\end{array}$ \\
\hline \multirow{2}{*}{ White } & -5 & 2.3572 & 2.3533 & 2.4485 & 2.1074 \\
& 0 & 2.22592 & 2.2925 & 2.2248 & 1.992 \\
& 5 & 2.6561 & 2.9417 & 2.6367 & 2.2573 \\
& 10 & 2.4769 & 2.6519 & 2.4244 & 2.0729 \\
& -5 & 2.5024 & 2.8985 & 2.8401 & 2.245 \\
Pink & 0 & 2.6802 & 2.9139 & 3.004 & 2.4468 \\
& 5 & 2.5809 & 2.5597 & 2.7417 & 2.2683 \\
& 10 & 2.3483 & 2.1798 & 2.3724 & 2.0895 \\
& -5 & 2.9137 & 2.8448 & 2.8678 & 2.6558 \\
& 0 & 2.5566 & 2.6244 & 2.5481 & 2.5113 \\
& 5 & 2.2415 & 2.2366 & 2.2284 & 2.1732 \\
& 10 & 1.9202 & 1.8551 & 1.8882 & 1.8487 \\
\hline
\end{tabular}


Table.2 Comparison of output PESQ of various threshold function

\begin{tabular}{|c|c|c|c|c|c|}
\hline Noise & $\begin{array}{c}\mathbf{S N R}_{\text {in }} / \\
\mathbf{d B}\end{array}$ & Document[7] & Document[13] & Document[15] & $\begin{array}{l}\text { Improved } \\
\text { threshold } \\
\text { function }\end{array}$ \\
\hline \multirow{4}{*}{ White } & -5 & 0.7884 & 1.435 & 1.5202 & 1.5792 \\
\hline & 0 & 1.2699 & 1.6698 & 1.6693 & 1.8124 \\
\hline & 5 & 1.9731 & 1.9853 & 1.8357 & 2.071 \\
\hline & 10 & 2.2386 & 2.2504 & 2.1074 & 2.3815 \\
\hline \multirow{4}{*}{ Pink } & -5 & 1.429 & 1.4481 & 1.6207 & 1.6469 \\
\hline & 0 & 1.7078 & 1.6771 & 1.7194 & 1.8562 \\
\hline & 5 & 2.1105 & 2.0934 & 2.0182 & 2.1614 \\
\hline & 10 & 2.3991 & 2.4492 & 2.3435 & 2.4365 \\
\hline \multirow{4}{*}{ Babble } & -5 & 1.5704 & 1.6071 & 1.5944 & 1.5585 \\
\hline & 0 & 1.9082 & 1.875 & 1.9428 & 1.8921 \\
\hline & 5 & 2.2338 & 2.1911 & 2.2349 & 2.2358 \\
\hline & 10 & 2.5114 & 2.5226 & 2.5121 & 2.5323 \\
\hline
\end{tabular}

Table 1 and Table 2 show PESQ and LSD values of noisy speech signals with different signal-to-noise ratios under different background noise conditions. The larger the PESQ value, the better the denoising effect. On the contrary, the smaller the LSD value, the better the denoising effect. It can be seen from the table that the improved threshold function algorithm is better than other algorithms in White noise and Pink noise, especially in low input signal-to-noise ratio. The PESQ and LSE values are better than other threshold function algorithms in this paper. In the case of Babble noise, although PESQ is slightly lower than other algorithms, it is better than others in the logarithmic spectrum distance. It may be that the speech spectrum of Babble noise with similar spectrum will pollute the intelligibility of low frequency band more obviously. However, the overall effect of the proposed algorithm is better than that of other algorithms in these noisy environments. More useful speech signals are retained, and the quality and clarity of speech perception are improved to a certain extent, which provides ideal pure signals for further processing of speech signals.

\section{Conclusion}

The improved threshold function proposed in this paper avoids the problems of fixed deviation and discontinuity at the threshold generated in the wavelet transform, and combines with the Bayesian threshold acquisition method to solve the problem of constant thresholds for different layers. In this paper, we use pure speech signal to add Gaussian white noise with different signal-to-noise ratios and then use different threshold functions to denoise the simulation, and compare the denoising effects of different threshold functions from the signal-to-noise ratio and root mean square error. The results show that the improved threshold function in this paper has a better denoising effect than other threshold functions, which improves the overall speech quality after denoising and achieves the ideal speech denoising effect. For the problem of unobvious multi-channel coincidence noise optimization, future work can consider introducing the latest fitness dependent optimize algorithm (FDO) and Dynamic Cat Swarm Optimization algorithm to achieve better denoising effects. 


\section{Acknowledgement}

This work was supported in part by the National Natural Science Foundation of China subsidization project (51579047), the Natural Science Foundation of Heilongjiang Province (QC2017048), the National Defense Technology Fundamental Research Funds (JSHS2015604C002), the Natural Science Foundation of Harbin (2016RAQXJ077), the fundamental research funds for the central universities (HEUCF180407), and the Open Project Program of State Key Laboratory of Millimeter Waves (K201707).

\section{References}

[1] Goel P, Garg A. Developments in spectral subtraction for speech[J]. International Journal of Engineering Research and Application, 2012, 2 (1) :55-63.

[2] Qian Qiuliang, Dong Baowei. Application of improved wavelet threshold denoising in bearing fault diagnosis[J]. Data Communication, 2020, 3(4): 17-19.

[3] Zhao Qiang, Yang Yang, Wang Yu, Guo Changjiang. An improved wavelet threshold denoising method and its simulation [J]. Modern Computer, 2021(10): 126-128..

[4] El-Fattah M A A, Dessouky M I, Abbas A M. Speech enhancement with adaptive wiener filter[J]. International Journal of Speech Technology, 2014, 17 (1) :53-64.

[5] Ma Jiachen, Hu Jiajun, Ma Liyong. Zhang Yong, Xie Wei. Active noise control based on improved particle swarm algorithm [J] . Computer Application Research, 2015, 9: 35-39.

[6] U Rajendra Acharya, Hamido Fujita, Muhammad Adam, et al. Automated Characterization and Classification of Coronary Artery Disease and Myocardial Infarction by Decomposition of ECG signals:A comparative Study[J].Information Sciences, 2017, 377:17-29.

[7] Galiano G, Velasco J.Rearranged Nonlocal Filters for Signal Denoising[J].Mathematics and Computers in Simulation, 2015, 118:213-223.

[8] Zhentao Yu, Jie Chen, Suqin Xu, Cheng Chi,Tingting Li. Wavelet-based Adaptive Enhancement Method of Aeromagnetic Anomaly Signal[J]. IOP Conference Series: Earth and Environmental Science, 2019, 237(3):032017 (6pp).

[9] Manuel Vilares Ferro,Víctor M. Darriba Bilbao,Jesús Vilares Ferro. Adaptive scheduling for adaptive sampling in pos taggers construction[J]. Computer Speech \& Language, 2020,60:

[10] LU Y, Huang YM, Xue W, Zhang GB. Seismic data processing is based on wavelet transform method to denoise [J]. Cluster calculation, 2019, 22 (3): 6609-6620. DOI: 10.1007/s10586-018-2355 -0.

[11] Du Shiqiang, Song Yukun, Zhang Xuan, Zhang Duoli. An improved wavelet threshold denoising algorithm [J]. Microelectronics and Computer, 2021, 38(02): 40-46.

[12] Liu Fengshan, Lv Zhao, Zhang Chao, Wu Xiaopei. Research on Speech Enhancement Alogrithm Based on Modified Wavelet Threshold Function [J] . Journal of Signal Pro- cessing, 2016, 32(2):203-213.

[13] Han Wang, Wenxi Lu, Zhenbo Chang. Simultaneous identification of groundwater contamination source and aquifer parameters with a new weighted-average wavelet variable-threshold denoising method[J]. Environmental Science and Pollution Research, 2021, :1-16.

[14] Lu Yong. The application of improved wavelet threshold function in speech enhancement[J]. Information Technology and Network Security, 2019, 38(08): 38-41.

[15] Qin Aina, Dai Liang, Li Fei, Cao Weihua. A Speech Enhancement Algorithm Based on Improved Wavelet Threshold Function [J] . Journal of Hunan University:Nat- ural Sciences, 2015, 42( 4):136140.

[16] Wang Ze, Wan Feng, Wong Chiman, Zhang Liming. Adaptive Fourier Decomposition Dased ECG Denoising[J]. Computers in Biology and Medicine, 2016, 77:195-205.

[17] Zhan Zhan, Qin Huibin. Wavelet threshold denoising algorithm based on new threshold function[J]. Computer Technology and Development, 2019, 29(11): 47-51.

[18] Liu Tianyu, Wang Zhu. A multi-objective particle swarm optimization algorithm with diversity control[J]. Journal of Xidian University (Natural Science Edition), 2021,48(3):106-114. DOI:10.19665/j.issn10012400.2021.03.014. 